\title{
Evaluation of the direct costs of epilepsy in a sample of Egyptian patients following up in Ain Shams University Hospital
}

\author{
Salem Abdelhady, Hossam Shokri, Mai Fathy and mona Mokhtar wahid el din * (D)
}

\begin{abstract}
Background: Epilepsy is a chronic disorder of the brain, and it is considered as the 4th common neurological disorder. Epilepsy is associated with an economic burden by imposing a significant burden on both the individuals who have the condition and on those around them, as well as for the society.

Objective: Our objective is to study and evaluate the direct costs associated with the medical management of epilepsy, one of the most common neurological conditions.

Methods: Observational, cross-sectional 4-month study started in December 2018 till April 2019 in patients between 2 and 60 years of age with epilepsy without other concomitant diseases. The direct medical costs include the number of neurology and emergency room visits, number and type of diagnostic tests, days of hospitalization, and treatment administered for epilepsy.

Results: We studied data from 194 patients, with a mean age of 30.6 years; the mean cost for treatment with carbamazepine was 78.6 Egyptian pound (EGP); for valproate, the cost was 288.4 EGP, and for levetiracetam, the cost was $491 \mathrm{EGP}$. The mean cost of electroencephalogram (EEG) is $108 \mathrm{EGP}$, and for computer tomography scan (CT) of the brain and magnetic resonance imaging (MRI) of the brain, it is 149.4 and 423.7 EGP respectively. The mean cost for hospitalization was 610.5 EGP, and the cost for intensive care unit (ICU) stay was 515.4 EGP.

Conclusion: The economic cost of epilepsy constitutes a major burden for the patients as well as their families; most of the direct cost was attributed to the purchase of AEDs. This necessitates creating strategies to ensure regular access to affordable AEDs as well as introducing other varieties of AEDs more potent with less side effects.
\end{abstract}

Keywords: Epilepsy, Economic burden, Antiepileptic cost

\section{Introduction}

Epilepsy is a chronic non-communicable disease of the brain that affects around 3.4 million individuals in the USA and 50 million people worldwide making the disease the 4th common neurological disorder, with nearly $80 \%$ of people with epilepsy live in low- and middle-income countries [1].

The overall lifetime prevalence of epilepsy was 7.60 per 1000 population and was higher in low-income

\footnotetext{
* Correspondence: Wahid_el_din@hotmail.com

Neurology Department, Faculty of Medicine, Ain Shams University, Cairo, Egypt
}

\section{Springer Open}

countries (8.75 per 1000) than in high-income countries (5.18 per 1000) [2]. In Egypt, a study done to estimate the prevalence of epilepsy in Al-Quseir found the prevalence to be 5.5/1000 [3], and another study done in AlManial Island found the prevalence to be 6.9/1000 [4].

Around 1/3 of patients with epilepsy live with uncontrolled seizures [5], which negatively impact their quality of life and the ability to function independently [6].

Medical care costs for epilepsy begin with the effort to detect underlying causes for seizures, to document cortical electrical abnormalities, and to initiate therapy with an antiseizure drug. This may involve outpatient clinic

(c) The Author(s). 2020 Open Access This article is licensed under a Creative Commons Attribution 4.0 International License, which permits use, sharing, adaptation, distribution and reproduction in any medium or format, as long as you give appropriate credit to the original author(s) and the source, provide a link to the Creative Commons licence, and indicate if changes were made. The images or other third party material in this article are included in the article's Creative Commons licence, unless indicated otherwise in a credit line to the material. If material is not included in the article's Creative Commons licence and your intended use is not permitted by statutory regulation or exceeds the permitted use, you will need to obtain permission directly from the copyright holder. To view a copy of this licence, visit http://creativecommons.org/licenses/by/4.0/. 
visits, emergency room visits, hospitalization, and the use of diagnostic evaluations such as electroencephalogram (EEG), computer tomography scans (CT), and magnetic resonance imaging (MRI). Follow-up treatment involves regular physician visits to monitor blood levels and to assess any side effects of the medications (leukocyte count and liver functions). If adequate control is not achieved with the first medication used, usually additional agents are tried. A small percentage of individuals who do not respond to multiple drug treatment may be considered for surgery or other therapies such as the ketogenic diet or vagal nerve stimulation [7].

In 2013, Cramer and colleagues estimated the annual cost of epilepsy per person in the USA to be 15,414 US dollars (\$), $20 \%$ for the use of medications, $38 \%$ for the outpatient related visits, and $24 \%$ for inpatients and emergency department visits. Moreover, the annual cost of epilepsy increases with an average of $\$ 9399$ greater in patients with uncontrolled epilepsy compared to patients with controlled epilepsy.

Epilepsy is associated with an economic burden by imposing a significant burden both on the individuals who have the condition and on those around them, as well as for the society.

The need to estimate the economic cost of epilepsy in a developing country like Egypt is to plan effective treatment with easy access without restriction for antiseizure drugs which represents an important potential avenue in which improvements may lead to lower costs, manage resources, highlight the importance of such a disease, and assess the importance of prevention.

Our objective is to study and evaluate the direct costs associated with the medical management of epilepsy, one of the most common neurological conditions.

\section{Methods}

This is an observational cross-sectional 12-month study that started in December 2018 till December 2019 of patients coming to the Epilepsy clinic in Ain Shams University hospital. It is a tertiary educational institution, managed by the Faculty of Medicine, serving around 2 million patients/year.

The study age ranges between 2 and 60 years of age with a diagnosis of epilepsy according to the International League Against Epilepsy (ILAE) 2017 criteria [8]. To ensure that the costs were attributable to epilepsy, patients with concomitant conditions (strokes, intracranial hemorrhage, tumors, degenerative diseases, metabolic conditions, and/or mental retardation) that could affect its course, therapeutic protocols, or the impact of treatment were excluded.

According to ILAE, epilepsy is defined by at least 2 unprovoked (or reflex) seizures occurring at least $24 \mathrm{~h}$ apart. Seizures are categorized by the onset into focal- onset seizures, generalized onset seizures, and seizures of unknown onset.

In our study, we considered patients with controlled epilepsy if they had not had seizures of any type for a minimum of 1 year while receiving the same dose of antiseizure drug or while not taking any medication [9], and since there is no agreement upon the definition of medically uncontrolled epilepsy, so we considered patients who had seizures in the last year to be uncontrolled.

Our study is a descriptive analysis of the direct cost of epilepsy which includes treatment with antiseizure medications, healthcare services as emergency care, hospital admissions, ICU care and consultation services, and diagnostic tests performed (basic blood test, antiseizure drug level, brain CT scan, brain MRI, EEG, long-term electroencephalogram, brain positron emission tomography (PET), brain single-photon emission computed tomography (SPECT)).

The cost of investigations and medication was referred to in Egyptian pounds (EGP) according to the price list from the Ain Shams University hospital, and we used the micro-costing (bottom-up) approach for costing of the health service, which depends on identifying and specifying all the resources that are used by each individual patient.

All procedures performed in studies involving human participants were in accordance with the ethical standards of the institutional and/or national research committee and with the 1964 Helsinki Declaration and its later amendments or comparable ethical standards.

Written informed consent was obtained from all individual participants involved in the study.

\section{Results}

We enrolled in our study a total of 194 epileptic patients, 104 males (53.6\%) and 90 females (46.4\%) (Table 1). The mean age was 30.6 years, with a standard deviation of 12.6; the youngest patient was 7 years old; and the oldest was 60 years old. The patients had been suffering from the disease for a mean of 12.9 years, with a standard deviation of 11.11; the longest duration of illness is 47 years, and the shortest is 1 year. Nine patients only showed high seizure frequency more than twice/month.

One hundred and fifty-eight patients $(81.4 \%)$ were diagnosed with generalized seizures and 36 (18.6\%) with focal seizures. Fifty-one patients (26.3\%) show good

Table 1 Gender distribution among patients

\begin{tabular}{lll}
\hline & Frequency & Percent \\
\hline Male & 104 & 53.6 \\
Female & 90 & 46.4 \\
Total & 194 & 100 \\
\hline
\end{tabular}


seizure control with no seizures in the last year of illness, and 143 patients $(87.6 \%)$ continued to experience seizures in the last year of illness and only 9 of them show high seizure frequency more than twice/month.

One hundred patients were on monotherapy (51.5\%), 79 of them (79\%) were taking classic antiseizure drugs (phenytoin, carbamazepine, or valproate) and 21 of them (21\%) were taking monotherapy new antiseizure drugs; 77 patients $(39.7 \%)$ were on polytherapy of antiseizure drugs, 64 of them $(83.11 \%)$ were on polytherapy of classic antiseizure drugs, 12 of them (15.5\%) were taking classic and new antiseizure drugs in polytherapy, and only one patient was taking polytherapy from new antiseizure drugs; and 17 patients $(8.8 \%)$ were either not receiving any medication or were non-compliant on medications (Table 2).

One hundred twenty-seven patients were using carbamazepine either as monotherapy or as polytherapy, consuming a total of 9986 tablets/month with a mean of 82.9 tablets/month and standard deviation of 40.9; as for valproate, there was a total of 87 patients using this medicine either as monotherapy or as polytherapy, consuming a total of 10037 tablets/month with a mean of 82.8 tablets/month and standard deviation of 42.8 . The use of other antiseizure drugs was limited in our patients as our hospital is used to dispense classic antiseizure drugs only like carbamazepine, valproate, and phenytoin; however, we had 22 patients on levetiracetam consuming a total of 1286 tablets/month with a mean of 58.4 tablets/month and standard deviation of 19.5 .

The mean cost for treatment with carbamazepine was 78.6 EGP; for valproate, the mean cost was 288.4 EGP; and for levetiracetam, the mean cost was 491 EGP.

Regular laboratory tests done for patients with epilepsy include complete blood picture and liver functions including aspartate aminotransferase and alanine aminotransferase. In our study, 132 patients (68\%) do these

Table 2 Types of antiseizure drug use

\begin{tabular}{lll}
\hline & Frequency & Percentage (\%) \\
\hline Monotherapy antiseizure drugs & 100 & 51.5 \\
$\begin{array}{l}\text { Monotherapy classic antiseizure drugs } \\
\text { (carbamazepine, phenytoin, valproate) }\end{array}$ & 79 & 40.7 \\
$\begin{array}{l}\text { Monotherapy new antiseizure drugs } \\
\text { (levetiracetam, topiramate, lamictal) }\end{array}$ & 21 & 10.8 \\
$\begin{array}{l}\text { Polytherapy antiseizure drugs } \\
\text { Polytherapy classic antiseizure drugs }\end{array}$ & 77 & 39.7 \\
$\begin{array}{l}\text { Polytherapy classic and new antiseizure } \\
\text { drugs }\end{array}$ & 12 & 33 \\
Polytherapy new antiseizure drugs & 1 & 6.2 \\
Non-compliance & 17 & 0.5 \\
Total & 194 & 100 \\
\hline
\end{tabular}

laboratory tests yearly and 62 patients (32\%) were either not doing these tests regularly or did not do it at all, with a mean cost of standard laboratory test of 54.4 $\mathrm{EGP} /$ patient/year. Regarding specific laboratory tests for medication serum level, 44 patients $(22.7 \%)$ do these tests yearly and 150 patients $(77.3 \%)$ are not, with a mean cost of 22.6 EGP/patient/year (Table 3).

EEG is one of the investigations used to diagnose and follow-up patients with epilepsy; our study shows 131 patients (58.2\%) do regular EEG once/year for follow-up and 81 patients $(41.8 \%)$ do not do regular EEG with a mean cost/patient of 108 EGP, 121 patients (62.4\%) did long-term EEG to help in diagnosis with a mean cost of 261.9 EGP, and 73 patients (37.6\%) did video EEG with a mean cost of 94 EGP. Regarding brain imaging either CT scan or MRI, our study shows 145 patients (74.7\%) did the scan at least once during their illness with a mean cost of 149.4 EGP and 137 patients (70.6\%) for the MRI with a mean cost of 423.7 EGP (Table 3).

Thirty-five patients did PET and/or SPECT either to identify non-specific lesions in the brain (in one patient, the brain MRI reported as low-grade glioma, and after PET scan, she was diagnosed with cortical dysplasia) or to detect temporal sclerosis. Overall, four patients only showed an abnormality in the scan, one diagnosed with cortical dysplasia and the other three with temporal sclerosis.

Most of our patients follow up regularly for refill medication with the mean number of outpatient clinic visits/month is 1.12 and the standard deviation is 3.049; however, the mean visits to the emergency department is 2.06 with a standard deviation of 8.10 and the mean number of hospitalizations per illness for each patient is 1.27 and the standard deviation is 5.67. Eighty-one patients (41.7\%) of our patient visit the emergency department at least once/year.

Epilepsy patients usually get hospitalized if they experienced repeated or uncontrolled seizures. In our

Table 3 Frequency of various epilepsy investigations

\begin{tabular}{ll}
\hline Investigations & $\begin{array}{l}\text { Patients who did the } \\
\text { investigation }(\boldsymbol{n}=\mathbf{1 9 4})\end{array}$ \\
\hline $\begin{array}{l}\text { Regular laboratory tests } \\
\text { (complete blood picture, } \\
\text { liver function tests) }\end{array}$ & $132(68 \%)$ \\
Antiseizure drug serum levels & \\
Regular EEG & $44(22.7 \%)$ \\
Video EEG & $131(68.2 \%)$ \\
Long term EEG & $73(37.6 \%)$ \\
Brain CT & $121(62.4 \%)$ \\
Brain MRI & $145(74.7 \%)$ \\
\hline
\end{tabular}

EEG electroencephalogram, CT computer tomography scan, MRI magnetic resonance imaging 
study, the mean days for hospital stay was 2.97 days and the standard deviation is 13.8 , and the mean cost for hospital stay was 610.5 EGP per patient with a standard deviation of 2748.4 EGP. Mean days of intensive care stay was 1.07 and a standard deviation of 7.4, with a mean cost of 515.4 EGP and a standard deviation of 3703.3 EGP.

\section{Discussion}

Our study is a cross-sectional study aiming to evaluate the direct cost of epilepsy. It was conducted in Ain Shams University hospital.

Ain Shams University hospital supports investigations and treatment at reduced prices; moreover, it also provides medical consultation and inpatient services almost free of charge.

We obtained data from 194 patients, with a mean age of 30.6 years. We found a predominance of generalized epilepsy over focal epilepsy. Our patients had been diagnosed with epilepsy for a mean of 12.9 years.

In our study, $51 \%$ of our patients were receiving monotherapy and nearly $39.7 \%$ were on polytherapy. The percentage of monotherapy treatment in our sample was low compared to the data from other studies [9-11]. Although the basic approach for the treatment of epilepsy is the use of a single antiseizure drug in order to maintain medication adherence, to avoid adverse effect, and to reduce the risk of interactions with other medications, our lower percentage of patients on monotherapy may be attributed to the availability of only 3 types of classical antiseizure drugs at our hospital (phenytoin, carbamazepine, and valproate) and the absence of other new-generation antiseizure drugs. The latter may as well explain the high percentage $(87.6 \%)$ of our patients with uncontrolled seizures having more than one seizure/year.

In terms of the type of medications used, most of our patients on monotherapy and on polytherapy were receiving classic antiseizure drugs. However, other studies showed that the most common polytherapy option was a combination of classic and new drugs [12, 13].

The mean cost for antiseizure drugs in our study differed according to the medicine used. For carbamazepine, the mean cost was $78.6 \mathrm{EGP} /$ month, representing an annual cost of 943.2 EGP. As for valproate, the mean monthly cost was 288.4 EGP, representing an annual cost of 3460 EGP. The burden of the cost of these medications is minimal on the patients, as these medications are fully financed by the hospital. On the other hand, levetiracetam, which is one of the new antiseizure drugs that is widely used in Egypt, is not provided for free by the hospital. The mean monthly cost of levetiracetam is 491 EGP, representing an annual cost of 5892 EGP.
Since different types of epilepsy respond to different antiseizure drugs and different individuals respond unpredictably differently as well to each drug, we may have to switch from one drug to another or to combine 2 or more drugs in order to reach a proper response. Because of the limited options provided by our hospital, the range of management procured to the patients is restricted, leading to an increase in the number of hospital visits (either emergency department or outpatient visits) and increased hospitalizations. This was obvious in our study as $41.7 \%$ of our patients at least visited the ER once/year, which is a very high ratio compared with other studies; Alison Kitson in 2000 reported that up to $18 \%$ of adults in the UK with active epilepsy reported attending the ER on the previous year. On the other hand, the mean days of hospital stay in our study was 2.97 and the mean intensive care stay was 1.07 days which was low compared to a study done in East China [14] in 2019 that stated that the median length of hospitalization was 13 days in patients with seizure disorder. Another study $[11,15]$, done in Germany, also reported a longer average duration for each epilepsycaused hospitalization (10-11.1 days). This contrast can be attributed to the fact that most epileptic patients in need of admission usually require to be hospitalized in the intensive care unit (ICU) or intermediate care unit. However, seeing that the number of ICU beds is limited all over the country, our hospital not being an exception, only a limited number of patients could be admitted to our facility, leading to the disparity between our findings and the findings of other studies.

The mean cost of investigations including blood tests, brain scanning, and electroencephalography per patient was 1037 EGP; added to them, the mean cost of medical visits plus hospitalizations was 1125.9 EGP. The mean annual cost is therefore 2162.9 EGP.

In our study, the price of antiseizure drugs has the greatest impact on direct medical costs and is considered the highest contributor to the direct cost. Our finding concurs with data from other developing countries where antiseizure drugs constitute a major expenditure in epilepsy management $[16,17]$. In spite of the fact that our hospital provides 3 classical antiseizure drugs for free, most of our patients have uncontrolled seizure, and this may be due to nonadherence to the medications because of the side effects, poor choices for antiseizure drug combinations, and/or non-availability of new antiseizure drugs. Poor adherence to medications can result in increased seizure frequency, less productivity, increased cost for managing comorbidities, greater social impact, and ultimately a multiplied economic burden.

The economic cost of epilepsy and the financial burden it constitutes for the community were high. This 
cost could be greatly reduced if the patient has easy access to investigations leading to early diagnosis and the availability of a wide range of antiseizure drug options for early seizure control.

To the best of our knowledge, our study is the first study to investigate the direct cost of epilepsy in Egypt.

\section{Conclusion}

The economic cost of epilepsy constitutes a major burden for the patients as well as their families; most of the direct cost was attributed to the purchase of antiseizure drugs. Therefore, strategies to ensure regular access to affordable antiseizure drugs, as well as introducing other varieties of antiseizure drugs more potent with less side effects, will not only cut down direct cost, but will also ensure better health outcomes and quality of life.

\section{Limitations}

We had some limitations in our study as our findings estimate the cost of epilepsy in Ain Shams University hospital and the cost of investigations and medications varies widely from one health care provider to another across Egypt and there is no fixed price for investigations or medications.

We estimated the direct cost of epilepsy only, and we did not cover the indirect cost, the quality of life, or the socioeconomic impact.

Our study is a descriptive study for the cost of epilepsy, and we did not do any comparative data as there is a lack of similar studies in our region.

All health care services at our facility are provided in lower prices for educational purposes compared to other facilities, so our estimate represents the lowest cost.

Also, we did not include in our study the medical cost of epilepsy-related complications like trauma for example.

\section{Abbreviations}

EEG: Electroencephalogram; ICU: Intensive care unit; CT: Computer tomography scan; MRI: Magnetic resonance imaging; $\$$ : US dollars; PET: Brain positron emission tomography; SPECT: Brain single-photon emission computed tomography; EGP: Egyptian pound

\section{Acknowledgements}

Not applicable

\section{Authors' contributions}

SA: designed and conceptualized the study, analyzed the data, and drafted the manuscript for intellectual content. HS: data collection and analysis including statistical analysis, drafting, and revision of the manuscript. MF: drafting and revision of the manuscript. MMW: designed and conceptualized the study, analyzed the data, and drafted the manuscript for intellectual content. All authors have agreed to conditions noted on the Authorship Agreement Form and have read and approved the final version submitted.

Funding

Self-financing.

\section{Availability of data and materials}

All data and materials that support findings in this manuscript are available. Please contact the corresponding author for data requests.

\section{Ethics approval and consent to participate}

The procedures followed were in accordance with the ethical standards of the responsible committee on human experimentation and with the principles of the Helsinki Declaration. Written informed consent was obtained from all participants and ethical committee permission from the Neuropsychiatry department of Ain Shams University on 18 October 2018.

\section{Consent for publication}

Not applicable

\section{Competing interests}

The authors declare that they have no competing interests.

Received: 25 June 2020 Accepted: 17 November 2020

Published online: 26 November 2020

\section{References}

1. Word Health Organization (2019) Epilepsy. https://www.who.int/news-room/ fact-sheets/detail/epilepsy.

2. Fiest KM, Sauro KM, Wiebe S, Patten SB, Kwon CS, Dykeman J, et al. Prevalence and incidence of epilepsy: a systematic review and meta-analysis of international studies. Neurology. 2017:88(3):296-303.

3. El Tallawy HN, Farghaly WM, Rageh TA, Shehata GA, Metwally NA, Badry R, et al. Spectrum of epilepsy - prevalence, impact, and treatment gap: an epidemiological study from Al-Quseir, Egypt. Neuropsychiatr Dis Treat. 2016; 12:1111-8.

4. Hashem S, Al-Kattan M, Ibrahim SY, Shalaby NM, Shamloul RM, Farrag M. Epilepsy prevalence in Al-Manial Island, Egypt. A door-to-door survey. Epilepsy Res. 2015;117:133-7.

5. Chen Z, Brodie MJ, Liew D, Kwan P. Treatment outcomes in patients with newly diagnosed epilepsy treated with established and new antiepileptic drugs: a 30-year longitudinal cohort study. JAMA Neurol. 2018;75(3):279-86.

6. Gupta S, Kwan P, Faught E, Tsong W, Forsythe A, Ryvlin P. Understanding the burden of idiopathic generalized epilepsy in the United States, Europe, and Brazil: an analysis from the National Health and Wellness Survey. Epilepsy Behav. 2016;55:146-56.

7. Marks WJ, Garcia PA. Management of seizures and epilepsy. Am Fam Physician. 1998;57:1589-600.

8. Fisher RS, Cross JH, D'Souza C, French JA, Haut SR, Higurashi N, et al. Instruction manual for the ILAE 2017 operational classification of seizure types. Epilepsia. 2017;58:531-42.

9. Kwan P, Brodie M. Early identification of refractory epilepsy. N Engl J Med. 2000;342(5):314-31.

10. Kwan P, Brodie MJ. Epilepsy after the first drug fails: substitution or add-on? Seizure. 2000;9:464-8.

11. Nodaa A, Hermsena A, Berkenfeld R, Dennigd D, Endrasse G, Kaltofenf J, et al. Evaluation of costs of epilepsy using an electronic practice management software in Germany. Seizure. 2015;26:49-55.

12. Beghi E, Garattini L, Ricci E, Cornago D, Parazzini F, EPICOS Group. Direct cost of medical management of epilepsy among adults in Italy: a prospective cost-of-illness study (EPICOS). Epilepsia. 2004;45:171-8.

13. Abasolo-Osinag E, Abecia-Inchaurregui LC, Etxeandia-Ikobaltzeta I, BurgosAlonso N, García-del Pozo J. A pharmaco epidemiological study of antiepileptic drug consumption (1992-2004). Rev Neurol. 2008;46:449-53.

14. Yu N, Lin X-J, Zhang S-G, Di Q. Analysis of the reasons and costs of hospitalization for epilepsy patients in East China. Seizure. 2019;72:40-5.

15. Thomas SV, Sarma PS, Alexander M, Pandit L, Shekhar L, Trivedi C, et al. Economic burden of epilepsy in India. Epilepsia. 2001;42:1052-60.

16. Nsengiyumwa G, Druet-Cabanac $M$, Nzisabira L, Preux PM, Vergnenègre $A$. Economic evaluation of epilepsy in Kiremba (Burundi): a case-control study. Epilepsia. 2004:45:673-7.

17. Pato-Pato A. Evaluation of the direct cost of epilepsy. J Neurol Neurosci. 2013;4:1-3.

\section{Publisher's Note}

Springer Nature remains neutral with regard to jurisdictional claims in published maps and institutional affiliations. 